Article

\title{
Modeling and Validation of the Radial Force Capability of Bearingless Hysteresis Drives
}

\author{
Salvatore Circosta *(D), Renato Galluzzi $(\mathbb{D}$, Angelo Bonfitto $(\mathbb{D}$, Luis M. Castellanos $(\mathbb{D}$, \\ Nicola Amati and Andrea Tonoli \\ Department of Mechanical and Aerospace Engineering, Polytechnic of Turin, 10129 Turin, Italy; \\ renato.galluzzi@polito.it (R.G.); angelo.bonfitto@polito.it (A.B.); luis.castellanos@polito.it (L.M.C.); \\ nicola.amati@polito.it (N.A.); andrea.tonoli@polito.it (A.T.) \\ * Correspondence: salvatore.circosta@polito.it; Tel.: +39-011-090-6239
}

Received: 31 August 2018; Accepted: 28 September 2018; Published: 2 October 2018

check for updates

\begin{abstract}
The hysteresis motor technology combined with the magnetic suspension makes bearingless hysteresis drives very appealing for high- and ultra-high-speed applications. Such systems exploit the magnetic behavior of the rotor material to achieve mechanical torque, but the hysteresis can significantly influence the magnetic suspension performance. The literature so far has focused mainly on the motor investigation. On the bearing side, the design and the performance assessment have been carried out by neglecting the hysteresis phenomenon of the rotor material. In those cases, the hysteresis of the rotor material is negligible and hence it slightly affects the force generation. In a wider perspective, this paper intends to investigate the force capability of electromagnetic actuators based on materials of large magnetic hysteresis behavior. To this purpose, the proposed numerical model, based on the finite element method, accounts for the magnetic hysteresis. The experimental results confirm the validity of the modeling approach, thus providing a useful tool for the design as well as the investigation of such systems.
\end{abstract}

Keywords: bearingless drives; electric machines; hysteresis motors; Jiles-Atherton model; semi-hard magnetic materials

\section{Introduction}

Semi-hard magnetic materials (SHMMs) are of great interest for high- and ultra-high-speed rotating electric machines because of their elevated mechanical strength and advantageous magnetic features. The magnetic hysteresis loop of these materials can be activated to yield mechanical torque. This principle is narrowed nowadays to very few motor applications due to the limited power density with respect to other electric machine solutions. However, at high speed, the simple structure and rotordynamic features of hysteresis motors allow unmatchable power density [1,2]. The magnetic behavior of SHMMs can also be used to achieve levitating forces, leading to magnetic bearing applications. In most turbomachinery, levitating journals require an electric machine to provide rotation. Active Magnetic Bearings (AMBs) and electric motors have very similar construction. Therefore, they can be combined into one single device: the bearingless drive. Its main advantages are lower component count and more compact layout than common solutions with separate electric motor and bearing system [3-6]. Furthermore, magnetic levitation is an advantageous solution for high-speed applications. Attractive features for high-speed machines are bearing losses lower than in conventional ball or journal bearings, lubrication-free operation, low maintenance costs along with the possibility to control the dynamics of the hovering [4,7-9]. These features motivated a wide use of AMBs in industrial applications such as compressors, turbines and flywheels. Nowadays, the research effort focuses on the control and hardware improvement [10-14] along with the system identification $[15,16]$. 
Usually, the rotating part of magnetic bearings is made of soft magnetic material with high magnetic permeability, which leads to low reluctance of the magnetic path, as well as a fairly linear behavior in a large operational range.

Bearingless hysteresis drives are a novel field of research where very few works have been published in the last years. Noh et al. [17] propose the comparison between hysteresis and reluctance bearingless motors. The rotor robustness and easier manufacturing of hysteresis drives along with their inherently smooth torque make them clearly advantageous. From the motor perspective, the coated solid rotor induction machine is a valid solution for high speed applications [18]. Its drawback is related to the overheating due to the eddy currents, which is significant at high speed. Conversely, the magnetic hysteresis phenomenon enables synchronous operation with respect to the rotating magnetic field, thus making hysteresis drives advantageous with respect to the asynchronous motors. Moreover, the rotor strength allows for reaching higher peripheral speed limits when compared to permanent-magnet-equipped technologies [19] and induction motors [20].

The available literature on bearingless hysteresis drives focuses mostly on the torque generation. On the bearing side, the design and the performance assessment have been carried out by neglecting the hysteretic behavior of the rotor materia [17,21]. In those cases, this assumption is justified since the energy dissipation due to hysteresis is rather low. Imani-Nejad [20] investigated the force generation by considering the magnetic hysteresis of the material through a one-dimensional approach. The hysteresis does not affect significantly the bearing performance since the employed material (D2 steel) exhibits a poor hysteretic behavior. Furthermore, only the minor hysteresis loops are activated. Hence, the magnetic capability of the material is not fully exploited since the achieved magnetic flux densities are significantly below the saturation value.

Additional efforts have investigated the magnetic hysteresis effect on the force generation of reluctance actuators for high-precision applications. One-dimensional analyses can be found in [22,23], whereas 2D and 3D finite element (FE) approaches have been also proposed [24]. All of these works address the hysteresis phenomenon exclusively on soft magnetic materials. Therefore, the magnetic hysteresis slightly affects the actuator performance.

In literature so far, no work has been conducted to study the force capability of electromagnetic actuators based on materials of large magnetic hysteresis behavior. To fulfill this gap, this paper presents the force characterization of an electromagnetic actuator based on FeCrCo 48/5 SHMM. In contrast to the above-mentioned literature, the applied magnetic fields are significant and, consequently, the nonlinear hysteretic behavior of the material needs to be considered. This work intends to build and experimentally validate a FE model able to capture the force generation phenomenon on SHMM-based AMBs.

The present work is structured as follows. The experimental setup is introduced in Section 2. Section 3 presents the numerical modeling. For comparison purposes, a system with the journal made of soft magnetic material and another one based on the SHMM are presented. The latter adopts the Jiles-Atherton model to reproduce the hysteresis behavior of the FeCrCo 48/5 SHMM. Afterwards, the experimental procedure is described in Section 4. Finally, Section 5 shows the comparison between numerical results and experimental data for both of the systems. In this regard, the results achieved from the system based on the soft magnetic material are used to identify the stator inner diameter. Then, the results obtained from the system based on the SHMM validate the proposed numerical model and lead to important considerations on the behavior of such systems. Section 6 presents the conclusions.

\section{Experimental Setup}

The system adopted for the experimental characterization is illustrated in Figure 1a. It is equipped with a standard 8-pole radial actuator for AMB systems. The test rig consists of two aluminum plates connected through four compliant beams (element 9 in Figure 1b). The lower plate (10) is fixed on a seismic mass (1) and supports a mobile stage (3) moved along two orthogonal directions by means of a 
micro-metric planar positioning stage (2). The journal (6), which cannot spin, is mounted on the mobile stage to have the manual control on the air gap length. The upper plate (4) holds the stator of the 8-pole radial actuator (7), including four electromagnets made of laminated high permeability silicon steel. The rig is equipped with exchangeable non-laminated journals (6) of soft iron and FeCrCo 48/5 SHMM. For simplicity, only one electromagnet is powered to perform a one-side actuation. The measurements are carried out in static conditions, with the journal at a stand-still. Each electromagnet counts 106 turns split on two coils, one per each pole of the C-core. The upper plate is connected to the seismic mass (13) through an HBM S2 load cell (12), which has a maximum measurement error of $0.02 \mathrm{~N}$ on a full scale of $100 \mathrm{~N}$. A fastener (11) interfaces the load cell and the upper plate in order to tune the compression preload of the load cell. The air gap is measured by means of a Bently-Nevada 3300XL eddy current sensor (8) (Minden, NV, USA) which has a maximum measurement error of $0.025 \mathrm{~mm}$. The power stage consists of one H-bridge driven by a $20-\mathrm{kHz}$ PWM carrier and fed by a $24-\mathrm{V} \mathrm{DC}$ bus. The current in the electromagnet is controlled by means of a Proportional-Integrative (PI) controller implemented on a STM 32F405VG board. The bandwidth of the current controller is $500 \mathrm{~Hz}$, sufficient for performing steady-state experimental tests. An AMP25 Hall sensor, with an accuracy of $10 \mathrm{~mA}$, is used for the current feedback.

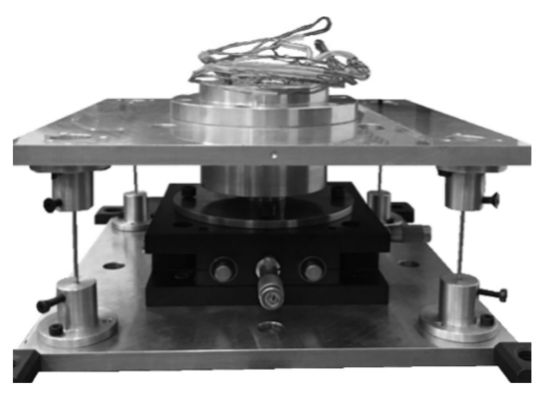

(a)

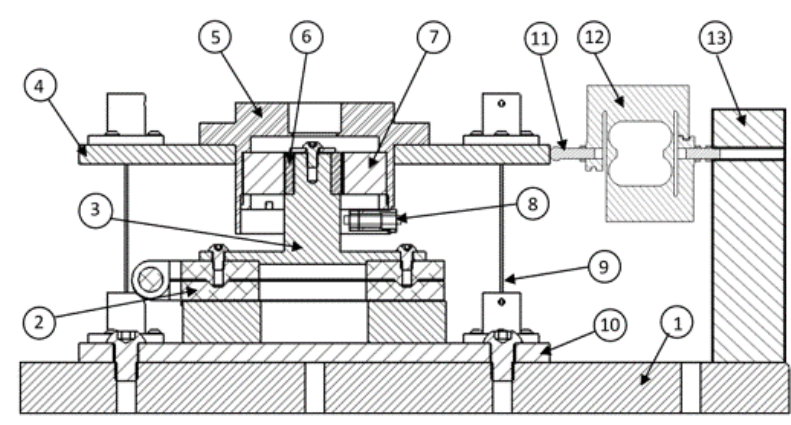

(b)

Figure 1. Experimental test rig (a); section view of the experimental setup (b): 1. Seismic mass; 2. Micrometric planar positioning stage; 3 . Support of the journal; 4 . Upper plate; 5 . Housing of the electromagnetic actuators; 6 . Journal; 7. Cores of the electromagnets; 8 . Proximitor sensor; 9. Flexible beams; 10. Lower plate; 11 . Load cell-upper plate interface; 12 . Load cell; 13. Seismic mass.

Table 1 resumes the main parameters of the electromagnetic actuators and the journals. Note that both the soft iron and the FeCrCo 48/5 SHMM journals have identical geometry. 
Table 1. Main parameters of the test rig.

\begin{tabular}{ccc}
\hline Symbol & Quantity & Value \\
\hline$A_{\text {pole }}$ & Polar area & $225 \mathrm{~mm}^{2}$ \\
$d_{r}$ & Journal inner diameter & $20.0 \mathrm{~mm}$ \\
$D_{r}$ & Journal outer diameter & $30.3 \mathrm{~mm}$ \\
$d_{s}$ & Stator inner diameter & $31.4 \mathrm{~mm}$ \\
$h$ & Journal height & $22.5 \mathrm{~mm}$ \\
$N$ & Turns per electromagnet & 106 \\
\hline
\end{tabular}

\section{Numerical Modeling}

The force generation phenomenon on the SHMM-based AMB is numerically investigated through the finite element method by means of a COMSOL Multiphysics model. The electromagnetic problem is formulated through the Magnetic Fields Interface under the AC/DC Module of the software solving the following electromagnetic problem formulation in terms of the magnetic vector potential $\mathbf{A}$ over a discretized domain

$$
\begin{aligned}
& \nabla \times \mathbf{H}=\mathbf{J}, \\
& \mathbf{B}=\nabla \times \mathbf{A}, \\
& \mathbf{J}=\sigma \mathbf{E}+\sigma \mathbf{v} \times \mathbf{B}+\mathbf{J}_{\mathbf{e}}, \\
& \mathbf{E}=-\frac{\partial \mathbf{A}}{\partial t}
\end{aligned}
$$

$\mathbf{H}$ being the magnetic field vector, $\mathbf{J}$ the current density, $\mathbf{B}$ the magnetic flux density vector, $\sigma$ the electrical conductivity, $\mathbf{v}$ the velocity of the conductor (which is null in the present analyses), $\mathbf{J}_{\mathbf{e}}$ an externally generated current density and $\mathbf{E}$ the electric field. This simulation can be executed either in stationary mode or, as in this study, in time-stepping mode. Further details about the implemented formulation can be found in [25].

The simulation tool requires boundary conditions and material constitutive relations to fully define the problem. The following nonlinear constitutive relation (whose reciprocal is taken from the software library and plotted in Figure 2), based on the nonlinear function $f_{F e}$, is adopted to consider the iron saturation in the static part of the actuator and the soft iron journal

$$
\mathbf{H}=f_{\mathrm{Fe}}(|\mathbf{B}|) \frac{\mathbf{B}}{|\mathbf{B}|} .
$$

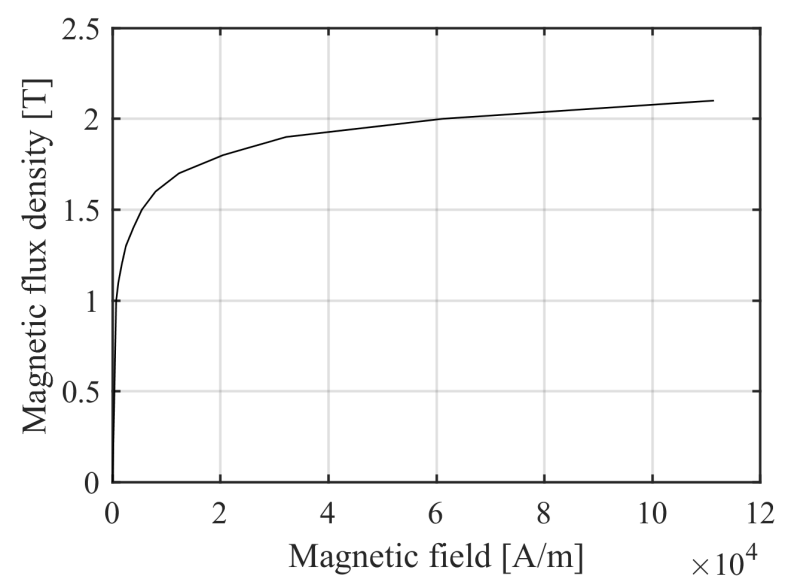

Figure 2. Magnetic characteristic of both the soft magnetic material and the laminated high permeability steel. 
The hysteretic behavior of the FeCrCo $48 / 5$ alloy is modeled through a vector generalization of the Jiles-Atherton model [26]. It is implemented in the software to describe the anisotropic magnetic behavior of the material. In this case, the magnetic constitutive relation is

$$
\mathbf{H}=\frac{1}{\mu_{0}} \mathbf{B}-\mathbf{M}
$$

where $\mu_{0}=4 \pi \cdot 10^{-7} \mathrm{H} / \mathrm{m}$ is the permeability of free space and $\mathbf{M}$ is the magnetization vector. It is calculated by solving the main equation of the Jiles-Atherton model

$$
\frac{\mathrm{d} \mathbf{M}}{\mathrm{d} t}=\mathbf{c} \frac{\mathrm{d} \mathbf{M}_{\mathrm{a}}}{\mathrm{d} t}+(\mathbf{I}-\mathbf{c}) \frac{\mathrm{d} \mathbf{M}_{\mathrm{i}}}{\mathrm{d} t}
$$

where $\mathbf{M}_{\mathrm{a}}$ and $\mathbf{M}_{\mathrm{i}}$ are the anhysteretic and the irreversible magnetization terms, respectively, $\mathbf{I}$ is the identity matrix and $\mathbf{c}$ is the reversibility tensor. For the $i$ th vector component, the Langevin function is used to express the anhysteretic behavior

$$
M_{\mathrm{a}, i}=M_{\mathrm{s}, i}\left(\operatorname{coth}\left(\frac{\left|\mathbf{H}_{\mathrm{e}}\right|}{a_{i}}\right)-\frac{a_{i}}{\left|\mathbf{H}_{\mathrm{e}}\right|}\right) \frac{H_{\mathrm{e}, i}}{\left|\mathbf{H}_{\mathrm{e}}\right|},
$$

where $M_{\mathrm{s}, i}$ is the saturation magnetization and $a_{\mathrm{s}, i}$ the Langevin slope for the $i$ th component. The effective magnetic field is

$$
\mathrm{H}_{\mathbf{e}}=\mathrm{H}+\alpha \mathbf{M}
$$

where $\alpha$ is the interdomain coupling tensor. The irreversible magnetization vector is obtained from the nonlinear equation

$$
\frac{\mathrm{d} \mathbf{M}_{\mathrm{i}}}{\mathrm{d} t}=\left[\left(\mathbf{k}^{-1} \mathbf{c}^{-1} \mathbf{M}_{\mathrm{r}}\right) \frac{\mathrm{d} \mathbf{H}_{\mathrm{e}}}{\mathrm{d} t}\right]^{+} \frac{\mathbf{k}^{-1} \mathbf{c}^{-1} \mathbf{M}_{\mathrm{r}}}{\left|\mathbf{k}^{-1} \mathbf{c}^{-1} \mathbf{M}_{\mathrm{r}}\right|}
$$

$\mathbf{k}$ being the pinning tensor and

$$
\mathbf{M}_{\mathrm{r}}=\mathbf{c}(\mathbf{c}-\mathbf{I})^{-1}\left(\mathbf{M}-\mathbf{M}_{\mathrm{a}}\right)
$$

the reversible magnetization vector.

The parameters needed to represent the radial and tangential characteristics (Table 2) have been identified by means of the least-squares optimization method proposed by Kis and Iványi [27]. The identification is based on the material radial and tangential hysteresis loops measured on FeCrCo 48/5 SHMM samples by means of a vibrating-sample magnetometer. Figure 3a,b show the comparison between the experimental and the numerical hysteresis loops.

Table 2. Jiles-Atherton tensor elements for anisotropic FeCrCo 48/5.

\begin{tabular}{ccc}
\hline Symbol & Quantity & Value \\
\hline$\alpha_{x x}, \alpha_{z z}$ & Local field factor (radial) & 0.16 \\
$c_{x x}, c_{z z}$ & Domain rotation loss (radial) & 0.69 \\
$a_{x x}, a_{z z}$ & Langevin parameter (radial) & $9.93 \times 10^{4} \mathrm{~A} / \mathrm{m}$ \\
$k_{x x}, k_{z z}$ & Pinning (radial) & $1.41 \times 10^{5} \mathrm{~A} / \mathrm{m}$ \\
$M_{S x x}, M_{S z z}$ & Saturation magnetization (radial) & $1.84 \times 10^{6} \mathrm{~A} / \mathrm{m}$ \\
$\alpha_{y y}$ & Local field factor (tangential) & 0.15 \\
$c_{y y}$ & Domain rotation loss (tangential) & 0.20 \\
$a_{y y}$ & Langevin parameter (tangential) & $9.94 \times 10^{4} \mathrm{~A} / \mathrm{m}$ \\
$k_{y y}$ & Pinning (tangential) & $5.16 \times 10^{4} \mathrm{~A} / \mathrm{m}$ \\
$M_{S y y}$ & Saturation magnetization (tangential) & $2.02 \times 10^{6} \mathrm{~A} / \mathrm{m}$ \\
\hline
\end{tabular}




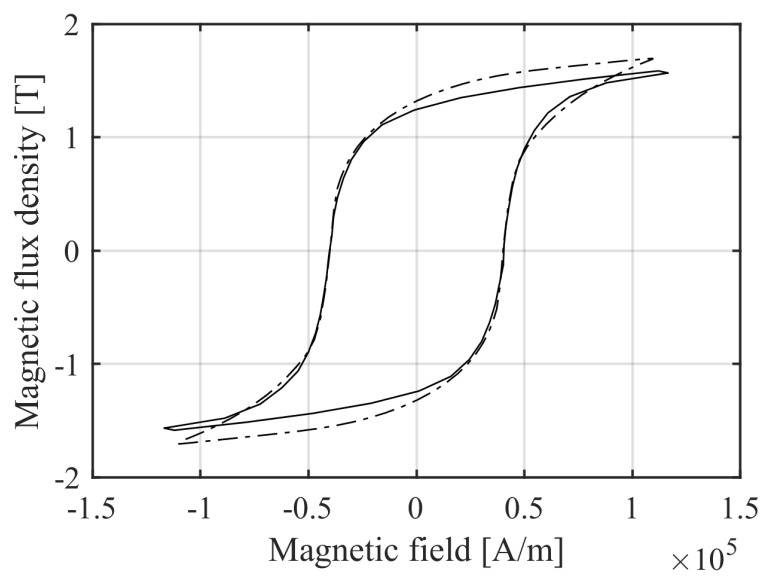

(a)

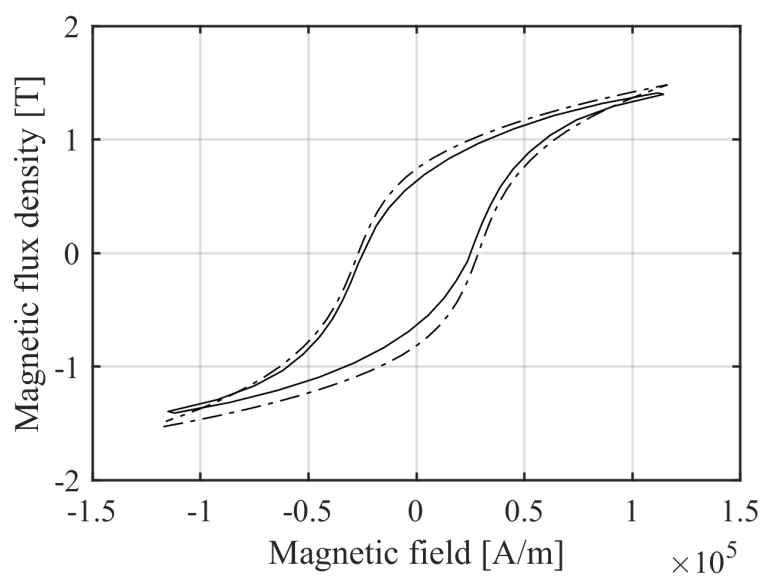

(b)

Figure 3. Radial (a) and tangential (b) magnetic characteristics of the $\mathrm{FeCrCo} 48 / 5$ alloy: experimental (solid); numerical (dash-dotted).

Both 2D and 3D finite element models are proposed. The 2D model (Figure 4a) assumes the vector potential with a single component normal to the plane of interest. This approach enables a significant reduction in the size of the problem but cannot consider possible effects of the end fields along the axial direction. The domain is discretized using quadratic triangular elements. The convergence study leads to the selection of the meshes constituted by 3438 elements for the model with the soft magnetic journal and 4420 elements for the one with the semi-hard magnetic journal (Figure $4 \mathrm{~b}$ ). The homogeneous Dirichlet's condition on the magnetic vector potential is applied to the external edge of the stator. It imposes all the magnetic flux lines tangent to the boundary. The coil is modeled as external current densities $J_{e}$ normal to the domains surrounding the stator poles as shown in Figure 4a.

The 3D model captures the effect of the end fields but is computationally more expensive than the $2 \mathrm{D}$ approach. It presents some issues in the convergence of the numerical solution. To improve the convergence robustness and obtain an efficient solving, linear tetrahedral elements are selected. Furthermore, symmetries of the geometry are exploited to reduce the size of the model. The symmetry with respect to the plane A (Figure 5a) is modeled by applying the homogeneous Dirichlet's condition on the component of the magnetic vector potential lying on the plane. The symmetry with respect to plane C (Figure 5a) is given by the homogeneous Neumann's condition on the component of the magnetic vector potential lying on the plane. It imposes the magnetic field perpendicular to the surfaces where the condition is applied. A further reduction of the model is achieved by considering that the magnetic flux is restricted in half of the model and does not cross the plane B (Figure 5a). 
This is modeled with the homogeneous Dirichelet's condition on the component of the magnetic vector potential lying on the plane B. Therefore, the model geometry is reduced to one eighth of its original size. The homogeneous Dirichlet's condition on the component of the magnetic vector potential lying on the boundary is applied to the external boundaries of the stator as well as the domain of air surrounding the tip of the magnetic bearing. The coil is modeled through the coil domain feature of the software. It implements a multi-wire coil by applying the main features of the coil (number of turns, wire cross-section, electrical conductivity of the material) to the interested domain. By defining the input and output boundaries of the coil, one preliminary analysis is needed to determine the local direction of the current flow in the domain. The convergence study leads to the selection of the meshes constituted by 44,906 elements for the model with the soft magnetic journal and 45,618 elements for the one with the semi-hard magnetic journal (Figure 5b).

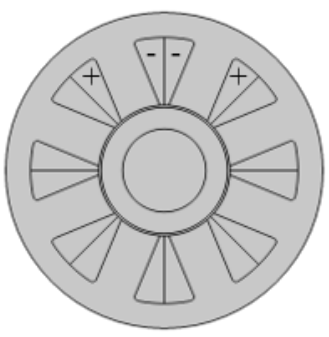

(a)

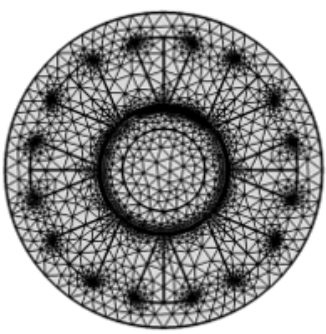

(b)

Figure 4. 2D finite element model: + and - refer to the domains where a positive and a negative current density $J_{e}$ is applied (a); meshed geometry of the system based on the semi-hard magnetic material (b).

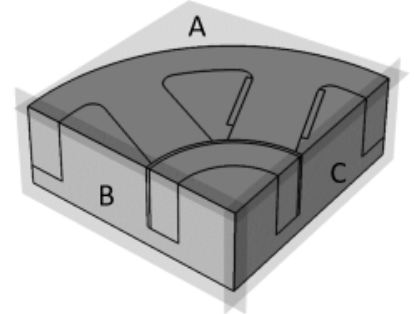

(a)

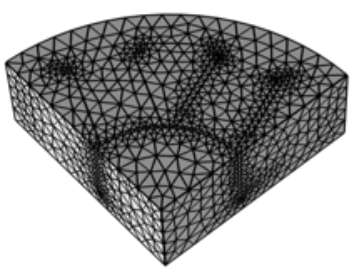

(b)

Figure 5. 3D finite element model showing the planes used to reduce the size of the numerical model (a); meshed geometry of the system based on the semi-hard magnetic material (b).

The generated force is computed by integrating the Maxwell's stress tensor over the boundary surface of the journal domain. For the 3D case, this computation is proportionally corrected by a factor four to account for the model reduction due to symmetry.

Since the Jiles-Atherton model is available mainly for time-dependent studies, the analysis in time is carried out. The input current follows a slow profile to reproduce the quasi-static nature of the test. Therefore, there is no dynamic effect in Equation (1) and the influence of the electrical conductivity becomes negligible.

A modified version of the proposed model can be adopted to conduct a complete dynamic analysis. In this case, the main dynamic contribution is related to the SHMM losses. Eddy current effects are already accounted by the time stepping solver, as seen in Equation (1). In addition, rate-dependent hysteresis models [28] can be implemented in COMSOL Multiphysics either through partial differential equations or as a C-compiled external material. More details regarding these two implementation methods can be found in the software manual [25]. 


\section{Test Procedure}

The test procedure is the same for both the setups with the soft and the semi-hard magnetic journals. Some preliminary steps are needed before the test:

1. The system is preloaded by unscrewing the fastener interfacing the load cell and the upper plate. This operation causes a relative displacement between the upper and the lower plates. Therefore, the preload is produced by the compliant beams.

2. The journal is moved by means of the sliding micrometer to approach the actuator edge. This position is identified as soon as the preload changes.

3. The journal is moved through the sliding micrometer to set the desired nominal air gap. The output of the eddy current sensor is recorded as reference.

After this preliminary procedure, the actuator coils are powered to achieve the needed current step-profile. The current is varied by small steps: each current step leads to a force variation $d F$ which causes a change in the air gap because of the load cell compliance. Hence, the nominal air gap has to be restored: the journal is moved by a quantity $d x$ through the sliding micrometer to restore the reference output of the eddy current sensor. Note that, after this operation, the relative position between the upper and the lower plates has changed by a quantity $d x$ and, consequently, the preload is different with respect to the previous current step. Now, the force measured by the load cell $F_{l c}$ is read, so the electromagnetic net force $F_{a c t}$ is computed:

$$
F_{a c t}^{i}=F_{l c}^{i}-F_{p l}^{i}
$$

in which the superscripts $i$ and $i-1$ refer to the present and the previous current steps, respectively. $F_{p l}$ is the net preload that considers the change in the relative position $d x$ between the upper and the lower plates, which is the quantity the journal needs to be moved through the sliding micrometer to restore the nominal air gap:

$$
F_{p l}^{i}=F_{p l}^{i-1}-k d x^{i}
$$

where $k$ is the mechanical stiffness of the compliant beams. It has been identified by manually varying the preload and measuring the resulting displacement through the eddy current sensor. The identified stiffness is $59.8 \mathrm{~N} / \mathrm{mm}$. Note that a current increase leads to $d F>0$, which, in turn, yields $d x<0$ and thus the preload decreases. The opposite is true if the current decreases.

Then, the current can be varied for the next current step. At the end of the test, the journal is degaussed through an alternating magnetic field with decreasing amplitude, achieved by supplying the actuator coil with a $25 \mathrm{~Hz}$ AC current with $5 \mathrm{~A}$ initial amplitude. This step is needed to start the successive test with no residual magnetization, which would have a significant influence in the case of the semi-hard magnetic journal.

\section{Results}

The system with the soft magnetic journal is investigated firstly. The numerical-experimental comparison of the force produced at $0.55 \mathrm{~mm}$ air gap is reported in Figure 6. The experimental data are achieved by increasing the current from 0 to $4 \mathrm{~A}$. The maximum current is limited by the mechanical instability of the test rig. This occurs because the mechanical stiffness that the load cell and the compliant beams provide is not sufficient to balance the negative stiffness introduced by the electromagnet. The numerical results are obtained with an input current ramp from 0 to 4 A. At first, the geometric air gap is considered. The comparison between $2 \mathrm{D}$ and $3 \mathrm{D}$ results shows that there is no influence of the end-field effects; therefore, the 2D model can be used for further analyses. The model with the geometric air gap overestimates the experimental force. This is due to the difference between the geometric and the magnetic air gap, which can be completely attributed to the stator [29]. In order to achieve the best fit, the stator inner diameter is increased by $0.06 \%$ to $31.42 \mathrm{~mm}$ (the identified non-magnetic layer is $0.02 \mathrm{~mm}$ ). 


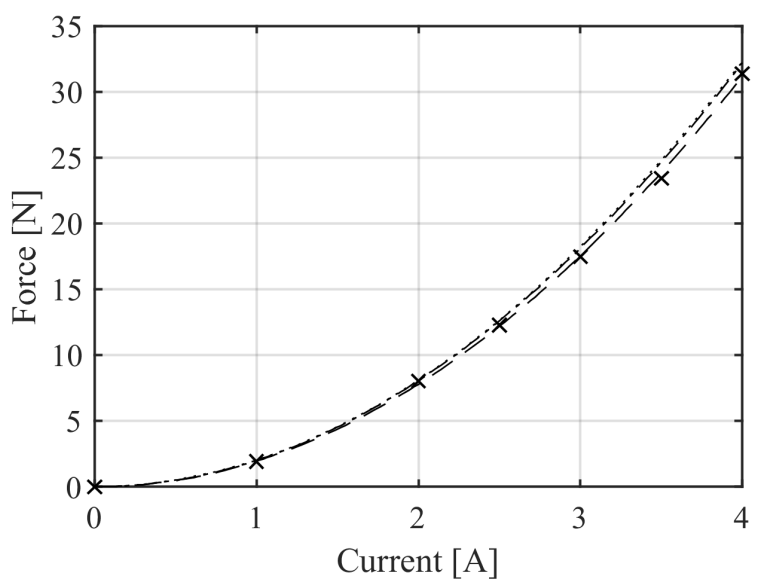

Figure 6. Numerical and experimental forces generated by the system based on the soft magnetic journal at $0.55 \mathrm{~mm}$ air gap: experimental data (cross); 2D model with magnetic air gap (dashed); 2D model with geometric air gap (dash-dotted); 3D model with geometric air gap (dotted). The dotted and dash-dotted lines are superimposed.

The experimental tests on the system based on the SHMM at three different air gap values $(0.55 \mathrm{~mm}, 0.50 \mathrm{~mm}$ and $0.45 \mathrm{~mm})$ are carried out by increasing the current from 0 to $6 \mathrm{~A}$ and then reducing it to $0 \mathrm{~A}$. The maximum current is limited to $6 \mathrm{~A}$ to avoid the overheating of the electromagnet coils. The numerical results consider the stator inner diameter identified through the analysis with the soft magnetic journal. Figure 7 shows the magnetic flux density distribution over the cross-section of the 3D model with $0.55 \mathrm{~mm}$ air gap at the maximum current. The contours of the normal component of the vector potential are plotted as well. These can be interpreted as magnetic flux lines and show how the flux is inclined to bypass the journal and tries to follow the shortest path across the journal. This is due to the low permeability of the $\mathrm{FeCrCo} 48 / 5$ and led to a quite unbalanced flux distribution in the air gap. The flux leakages are also responsible for the mismatch between the 2D and the 3D force prediction (Figure 8). To investigate more deeply this effect, the magnetic flux straying through the top face of one polar expansion $\Phi_{T}$ (Figure 9) is computed. It is compared to the magnetic flux $\Phi_{P}$ (Figure 9) flowing through the polar area, that is the flux responsible for the force generation. The ratio $\Phi_{T} / \Phi_{P}$ is plotted for the three different air gap values (Figure 10). It is observed that the flux leakage in axial direction is not negligible with respect to the useful flux, thus explaining why the 2D model overestimates the generated force. The ratio decreases at smaller air gaps since the flux paths crossing the journal imply a lower reluctance and, consequently, the leakage decreases. Figure 8 shows a comparison between the 3D force prediction and the experimental results at the three different air gaps. The maximum error of the predicted force at the different air gaps is reported in Table 3. The influence of the measurement error, which is related to the accuracy of the sensors (see Section 2), is negligible. The results show that the numerical model overestimates the experimental force. This behavior can be ascribed to the numerical representation of the FeCrCo 48/5. The Jiles-Atherton model is usually employed to represent the magnetic behavior of soft magnetic alloys, which exhibit a narrow coercive magnetic fields when compared to SHMMs. In such applications, this model is demonstrated to be accurate $[27,30,31]$. Conversely, in this case, the SHMM presents a high coercive field and the Jiles-Atherton approach does not perfectly reproduce the magnetic behavior of the material, as illustrated in Figure 4. This limitation can be ascribed to the pinning parameter $k$, which is assumed constant during the magnetization process $[28,30]$.

The influence of the magnetic hysteresis on the force generation of the SHMM-based system is evident. The difference between the loading and the unloading force profiles at the three air gap values (Figure 8) is significant. Note that after the tests the residual forces are $1.47 \mathrm{~N}, 2.08 \mathrm{~N}$ and $2.59 \mathrm{~N}$ at $0.55 \mathrm{~mm}, 0.50 \mathrm{~mm}$ and $0.45 \mathrm{~mm}$ air gap, respectively. By comparing the force generated by the systems based on the soft magnetic journal (Figure 6) and the $\mathrm{FeCrCo} 48 / 5$ journal (Figure 8a) at 
$0.55 \mathrm{~mm}$ air gap, it is clear that the SHMM magnetic behavior significantly lowers the force capability of the electromagnetic actuator. A quantitative analysis of this comparison is reported in Table 4 from $0 \mathrm{~A}$ to $4 \mathrm{~A}$ in loading and unloading conditions. If analyzed in loading conditions, the ratio between the two forces $\left(F_{s o f t} / F_{S H M M}\right)$ tends to be smaller at higher supplied currents mainly because of the above-mentioned flux leakage reduction in the SHMM electromagnet. On the other hand, the ratio in unloading conditions is lower than in the previous case because of the higher level of magnetization in the SHMM. These results demonstrate that the effects of previous magnetization in SHMMs represent a potential improvement in the force capability of the studied system. However, this aspect goes beyond the purpose of the present research.

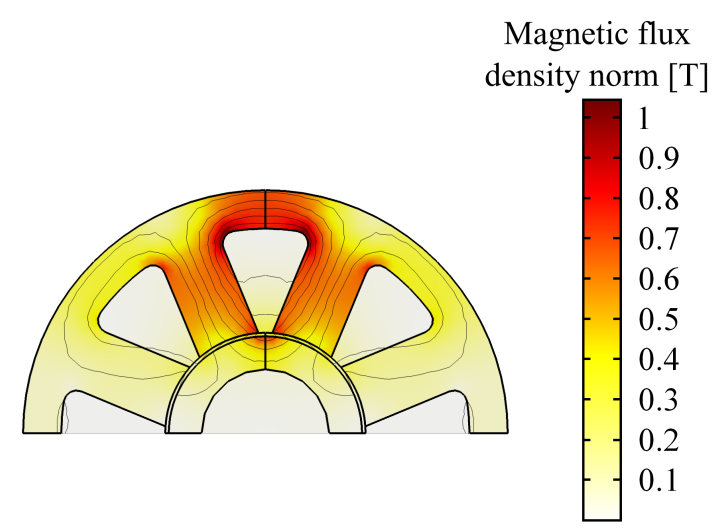

Figure 7. Magnetic flux density norm (surface plot) and magnetic vector potential (contour plot) over the cross-section of the 3D model based on the $\mathrm{FeCrCo} 48 / 5$ at $0.55 \mathrm{~mm}$ air gap and 6 A supplied current.

Table 3. Maximum relative error in the predicted force for the electromagnet based on FeCrCo 48/5.

\begin{tabular}{ccc}
\hline Air Gap [mm] & Current & Error \% \\
\hline 0.55 & 1 A-Unloading & 18 \\
0.50 & 2 A-Unloading & 19 \\
0.45 & 3 A-Unloading & 16 \\
\hline
\end{tabular}

Table 4. Ratio between the forces generated at $0.55 \mathrm{~mm}$ air gap by the electromagnet based on the soft and the semi-hard magnetic material, respectively.

\begin{tabular}{ccc}
\hline Current $[\mathrm{A}]$ & $\mathbf{F}_{\text {soft }} / \mathbf{F}_{\text {SHMM }}$ (Loading) & $\mathbf{F}_{\text {soft }} / \mathbf{F}_{\text {SHMM }}$ (Unloading) \\
\hline 1 & 6.9 & 0.6 \\
2 & 5.7 & 1.4 \\
3 & 4.8 & 2.0 \\
4 & 4.3 & 2.5 \\
\hline
\end{tabular}




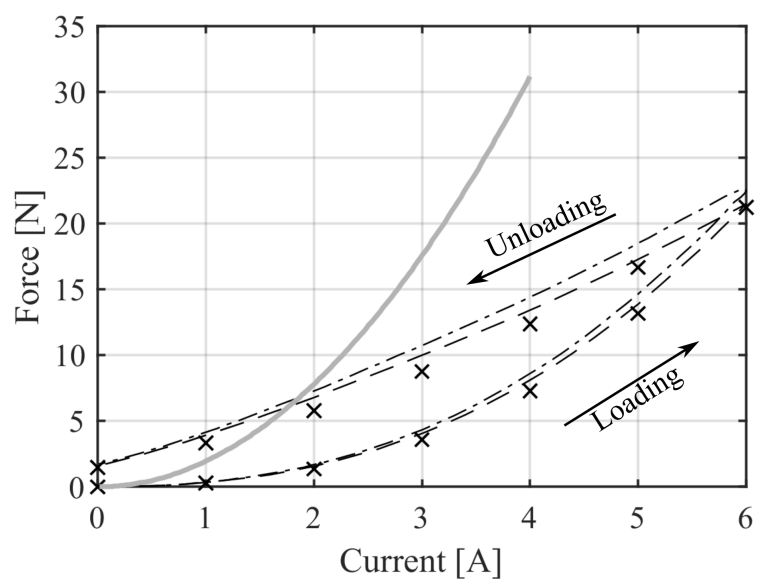

(a)

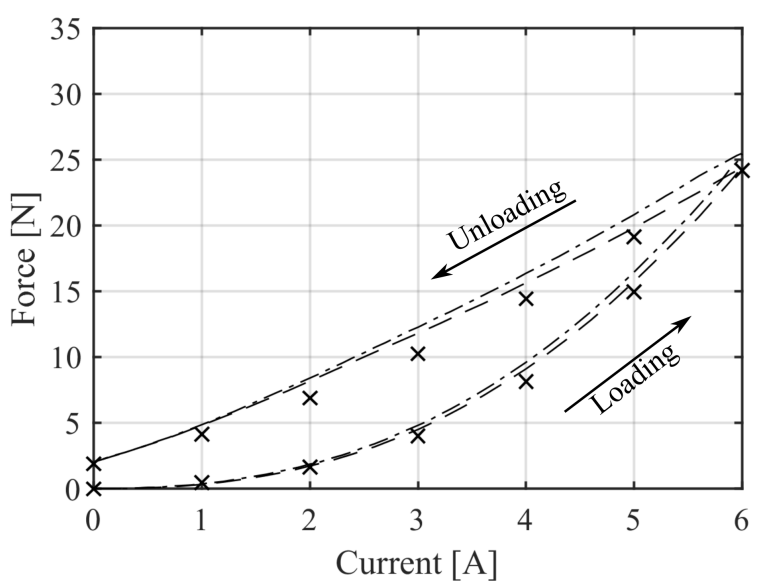

(b)

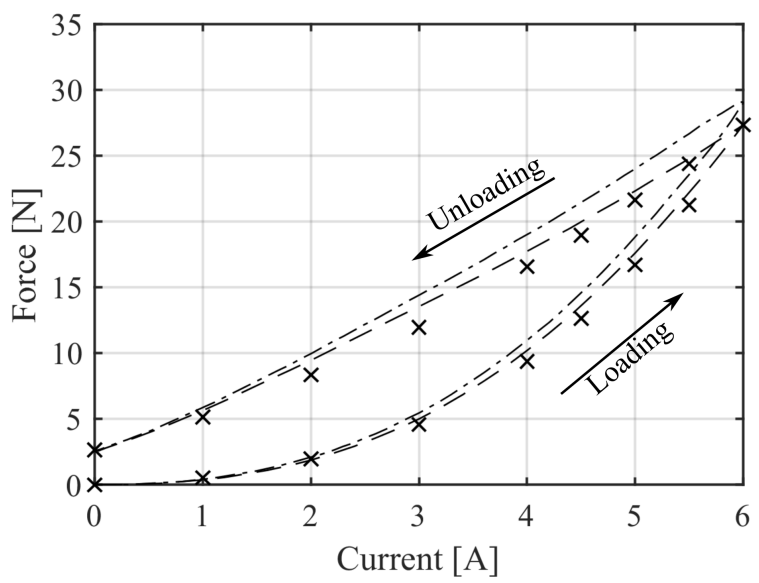

(c)

Figure 8. Numerical and experimental forces generated by the system based on the FeCrCo $48 / 5$ journal at $0.55 \mathrm{~mm} \mathrm{(a),} 0.50 \mathrm{~mm}(\mathbf{b})$ and $0.45 \mathrm{~mm}$ (c) air gap: experimental data (cross); 3D model with magnetic air gap (dashed); 2D model with magnetic air gap (dash-dotted). Loading and unloading curves are related to the current increase from 0 to $6 \mathrm{~A}$ and the current decrease from 6 to $0 \mathrm{~A}$, respectively. The grey solid line in (a) is the force achieved from the 2D model with magnetic air gap (same as Figure 6). 


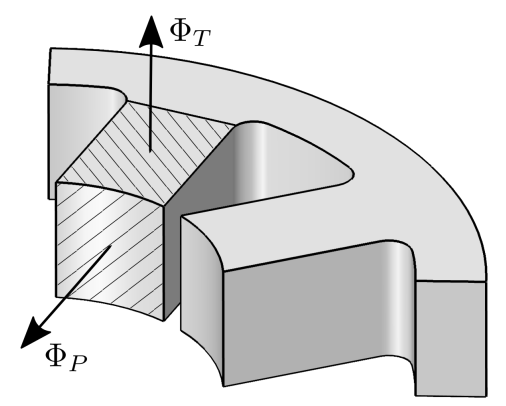

Figure 9. One quarter of the stator of the radial 8-poles radial actuator: $\Phi_{T}$ is the magnetic flux straying through the top face of one polar expansion; $\Phi_{P}$ is the magnetic flux flowing through the polar area.

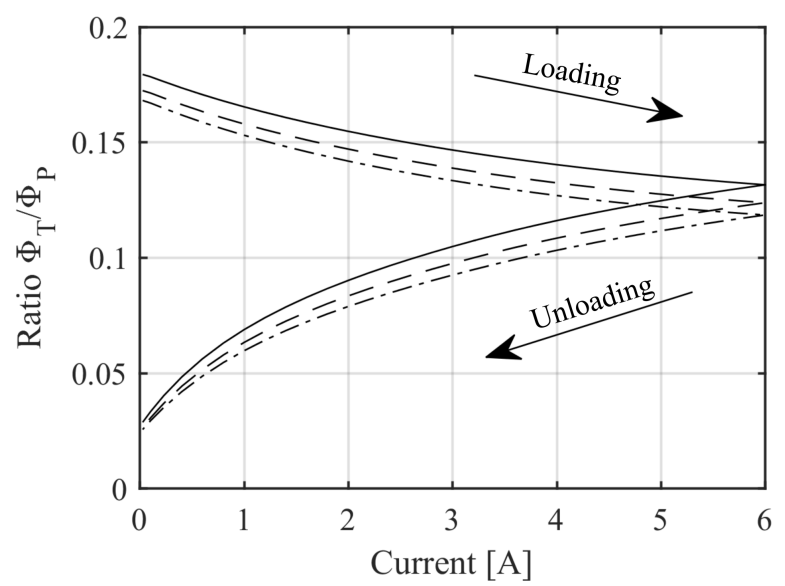

Figure 10. Ratio between the magnetic flux through the top face of one polar expansion of the electromagnet and the flux flowing through the polar area at $0.55 \mathrm{~mm}$ (solid), $0.50 \mathrm{~mm}$ (dashed) and $0.45 \mathrm{~mm}$ (dash-dotted) air gap for the system based on the FeCrCo 48/5 journal. Loading and unloading curves are related to the current increase from 0 to 6 A and the current decrease from 6 to 0 A, respectively.

\section{Conclusions}

This paper presented the FE modeling and validation of electromagnetic actuators based on SHMM to assess their force capability. At first, the stator inner diameter was identified by means of the numerical-experimental comparison of the force generated by the system based on the soft magnetic journal. Then, the FE model of the system based on the FeCrCo $48 / 5$ journal was validated. The numerical results show that the flux leakage is a delicate aspect and needs to be accurately considered in the design of such electromagnetic actuators. Furthermore, the unbalanced flux distribution in the air gap makes the force capability difficult to be addressed through 1D approaches. Therefore, the proposed tool is demonstrated to be valid in the design of electromagnetic actuators exploiting magnetic hysteresis. From the perspective of the bearingless hysteresis motor, the $\mathrm{FeCrCo}$ $48 / 5$ alloy is advantageous because of its large magnetic energy density, which raises the torque capability. Conversely, the main drawback is that the magnetic behavior of the SHMM significantly affects the force output of the system.

Author Contributions: Conceptualization, S.C., R.G., A.B., N.A. and A.T.; Investigation, R.G. and A.B.; Methodology, Salvatore Circosta; Software, L.M.C.; Supervision, N.A. and A.T.; Validation, Salvatore Circosta and L.M.C.; Writing_-Original draft, S.C.; Writing—Review and editing, R.G., A.B., N.A. and A.T.

Funding: This research received no external funding. 
Acknowledgments: The authors would like to thank Massimo Pasquale and his team at Istituto Nazionale di Ricerca Metrologica (INRiM) for helping with the magnetic characterization of the FeCrCo 48/5 alloy.

Conflicts of Interest: The authors declare no conflict of interest.

\section{References}

1. Galluzzi, R.; Tonoli, A.; Amati, N. Magnetic hysteresis machines for next-generation electric turbochargers. In Proceedings of the 2017 International Conference of Electrical and Electronic Technologies for Automotive, Torino, Italy, 15-16 June 2017; pp. 1-5. [CrossRef]

2. Nasiri-Zarandi, R.; Mirsalim, M.; Tenconi, A. A Novel Hybrid Hysteresis Motor With Combined Radial and Axial Flux Rotors. IEEE Trans. Ind. Electr. 2016, 63, 1684-1693. [CrossRef]

3. Chiba, A. (Ed.) Magnetic Bearings and Bearingless Drives; OCLC: 179729376; Elsevier/Newnes: Amsterdam, The Netherlands; London, UK, 2005.

4. Bleuler, H.; Cole, M.; Keogh, P.; Larsonneur, R.; Maslen, E.; Okada, Y.; Schweitzer, G.; Traxler, A. Magnetic Bearings: Theory, Design, and Application to Rotating Machinery; Springer Science \& Business Media: Berlin, Germany, 2009.

5. Lin, F.C.; Yang, S.M. Self-Bearing Control of a Switched Reluctance Motor Using Sinusoidal Currents. IEEE Trans. Power Electr. 2007, 22, 2518-2526. [CrossRef]

6. Okada, Y.; Yamashiro, N.; Ohmori, K.; Masuzawa, T.; Yamane, T.; Konishi, Y.; Ueno, S. Mixed Flow Artificial Heart Pump with Axial Self-Bearing Motor. IEEE/ASME Trans. Mech. 2005, 10, 658-665. [CrossRef]

7. Lei, S.; Palazzolo, A. Control of flexible rotor systems with active magnetic bearings. J. Sound Vib. 2008, 314, 19-38. [CrossRef]

8. Baumgartner, T.; Burkart, R.M.; Kolar, J.W. Analysis and Design of a 300-W 500,000-r/min Slotless Self-Bearing Permanent-Magnet Motor. IEEE Trans. Ind. Electr. 2014, 61, 4326-4336. [CrossRef]

9. Filatov, A.; Hawkins, L.; McMullen, P. Homopolar Permanent-Magnet-Biased Actuators and Their Application in Rotational Active Magnetic Bearing Systems. Actuators 2016, 5, 26. [CrossRef]

10. Lusty, C.; Bailey, N.Y.; Keogh, P.S. Control of Flexible Rotor Vibration with Flexibly Mounted Active Magnetic Bearings. In Proceedings of the 10th International Conference on Rotor Dynamics-IFToMM; Cavalca, K.L., Weber, H.I., Eds.; Springer International Publishing: Cham, Switzerland, 2019; pp. 65-73.

11. Bonfitto, A.; Botto, G.; Chiaberge, M.; Suarez Cabrera, L.; Tonoli, A. A multi-purpose control and power electronic architecture for active magnetic. In Proceedings of the 2012 15th International Power Electronics and Motion Control Conference (EPE/PEMC), Novi Sad, Serbia, 4-6 September 2012; p. DS2b.9-1.

12. Ferreira, J.; Maslen, E.; Fittro, R. Transpermeance Amplifier Applied to Magnetic Bearings. Actuators $2017,6,9$. [CrossRef]

13. Bonfitto, A.; Castellanos Molina, L.M.; Tonoli, A.; Amati, N. Offset-Free Model Predictive Control for Active Magnetic Bearing Systems. Actuators 2018, 7, 46. [CrossRef]

14. Bonfitto, A.; Tonoli, A.; Silvagni, M. Sensorless active magnetic dampers for the control of rotors. Mechatronics 2017, 47, 195-207. [CrossRef]

15. Castellanos, L.M.; Bonfitto, A.; Tonoli, A.; Amati, N. Identification of Force-Displacement and Force-Current Factors in an Active Magnetic Bearing System. In Proceedings of the 18th Annual IEEE International Conference on Electro Information Technology, Rochester, MI, USA, 3-5 May 2018.

16. Noshadi, A.; Shi, J.; Lee, W.S.; Shi, P.; Kalam, A. System Identification and Robust Control of Multi-Input Multi-Output Active Magnetic Bearing Systems. IEEE Trans. Control Syst. Technol. 2016, 24, 1227-1239. [CrossRef]

17. Noh, M.; Gruber, W.; Trumper, D.L. Hysteresis Bearingless Slice Motors with Homopolar Flux-Biasing. IEEE/ASME Trans. Mech. 2017, 22, 2308-2318. [CrossRef] [PubMed]

18. Gerada, D.; Mebarki, A.; Brown, N.L.; Gerada, C.; Cavagnino, A.; Boglietti, A. High-Speed Electrical Machines: Technologies, Trends, and Developments. IEEE Trans. Ind. Electr. 2014, 61, 2946-2959. [CrossRef]

19. Jagiela, M.; Bumby, J.; Spooner, E. Time-domain and frequency-domain finite element models of a solid-rotor induction/hysteresis motor. IET Electr. Power Appl. 2010, 4, 185-197. [CrossRef]

20. Nejad, M.I. Self-Bearing Motor Design \& Control. Ph.D. Thesis, Massachusetts Institute of Technology, Cambridge, MA, USA, 2013. 
21. Zhou, L.; Imani Nejad, M.; Trumper, D.L. One-axis hysteresis motor driven magnetically suspended reaction sphere. Mechatronics 2017, 42, 69-80. [CrossRef]

22. Liu, Y.P.; Liu, K.Z.; Yang, X. Nonlinear Current Control for Reluctance Actuator with Hysteresis Compensation. J. Control Sci. Eng. 2014, 2014, 1-7. [CrossRef]

23. Katalenic, A.; de Boeij, J.; Butler, H.; van den Bosch, P. Linearization of a current-driven reluctance actuator with hysteresis compensation. Mechatronics 2013, 23, 163-171. [CrossRef]

24. Vrijsen, N.H.; Jansen, J.W.; Lomonova, E.A. Prediction of Magnetic Hysteresis in the Force of a Prebiased E-Core Reluctance Actuator. IEEE Trans. Ind. Appl. 2014, 50, 2476-2484. [CrossRef]

25. COMSOL Multyphysics Reference Manual, version 5.3, COMSOL, Inc. Available online: www.comsol.com (accessed on 1 October 2018).

26. Bergqvist, A. A simple vector generalization of the Jiles-Atherton model of hysteresis. IEEE Trans. Magn. 1996, 32, 4213-4215. [CrossRef]

27. Kis, P.; Iványi, A. Parameter identification of Jiles-Atherton model with nonlinear least-square method. Phys. B Condens. Matter 2004, 343, 59-64. [CrossRef]

28. Szewczyk, R.; Frydrych, P. Extension of the Jiles-Atherton Model for Modelling the Frequency Dependence of Magnetic Characteristics of Amorphous Alloy Cores for Inductive Components of Electronic Devices. Acta Phys. Pol. A 2010, 118, 782-784. [CrossRef]

29. Antila, M.; Lantto, E.; Arkkio, A. Determination of forces and linearized parameters of radial active magnetic bearings by finite element technique. IEEE Trans. Magn. 1998, 34, 684-694. [CrossRef]

30. Jiles, D.C.; Atherton, D.L. Theory of ferromagnetic hysteresis (invited). J. Appl. Phys. 1984, 55, $2115-2120$. doi:10.1063/1.333582. [CrossRef]

31. Chwastek, K.; Szczyglowski, J. Identification of a hysteresis model parameters with genetic algorithms. Math. Comput. Simul. 2006, 71, 206-211. [CrossRef]

(C) 2018 by the authors. Licensee MDPI, Basel, Switzerland. This article is an open access article distributed under the terms and conditions of the Creative Commons Attribution (CC BY) license (http:/ / creativecommons.org/licenses/by/4.0/). 